\title{
Comparative Omics Analysis of Historic and Recent Isolates of Bordetella pertussis and Effects of Genome Rearrangements on Evolution
}

\author{
Ana Dienstbier, Fabian Amman, Denisa Petráčková, Daniel Štipl, Jan Čapek, Jana Zavadilová, \\ Kateřina Fabiánová, Jakub Držmíšek, Dilip Kumar, Mark Wildung, Derek Pouchnik, Branislav Večerek
}

Despite high vaccination coverage, pertussis is increasing in many industrialized countries, including the Czech Republic. To better understand Bordetella pertussis resurgence, we analyzed historic strains and recent clinical isolates by using a comparative omics approach. Whole-genome sequencing showed that historic and recent isolates of $B$. pertussis have substantial variation in genome organization and form separate phylogenetic clusters. Subsequent RNA sequence analysis and liquid chromatography with mass tandem spectrometry analyses showed that these variations translated into discretely separated transcriptomic and proteomic profiles. When compared with historic strains, recent isolates showed increased expression of flagellar genes and genes involved in lipopolysaccharide biosynthesis and decreased expression of polysaccharide capsule genes. Compared with reference strain Tohama I, all strains had increased expression and production of the type III secretion system apparatus. We detected the potential link between observed effects and insertion sequence element-induced changes in gene context only for a few genes.

Bordetella pertussis is a gram-negative, strictly huB man pathogen of the respiratory tract and the major causative agent of whooping cough. This highly contagious disease is especially severe in infants and

Author affiliations: Institute of Microbiology of the Czech Academy of Sciences, Prague, Czech Republic (A. Dienstbier, D. Petráčková, D. Štipl, J. Čapek, J. Držmíšek, D. Kumar, B. Večerek); Institute for Theoretical Chemistry of the University of Vienna, Vienna, Austria (F. Amman); National Institute of Public Health, Prague (J. Zavadilová, K. Fabiánová); Washington State University, Pullman, Washington, USA (M. Wildung, D. Pouchnik)

DOI: https://doi.org/10.3201/eid2701.191541 remains a major cause of infant illness and death worldwide, predominantly in industrialized countries (1). Although pertussis is a vaccine-preventable disease, increased incidence is being observed in some countries that have highly vaccinated populations, including the Czech Republic (2-4). Although several factors are contributing to pertussis resurgence in these countries (5-7), the 2 prominent factors are incomplete and short-lived immunity induced by current acellular vaccines (8-10) and genetic variation, leading to escape from immunity by antigenic variation (11-13).

$B$. pertussis has an efficient mechanism of genome structure diversification because it contains $>200$ copies of insertion sequence 481 (IS481) in its genome (14). IS element-mediated homologous recombination results in excision or insertion of flanking genome regions and leads to genome reduction and decay (14-16), as well as genome rearrangements $(17,18)$ and large duplications $(19)$. Furthermore, a previous study indicated that gene order rearrangements associated with IS elements can alter gene expression profiles in B. pertussis (20). Recently, we have shown that, besides their effect on genome structure and stability, ISs can affect expression profiles of neighboring genes by IS element-specific promoters (21).

On the basis of these observations, we hypothesized that strains with different genomic organization should display altered global transcriptomic and, consequently, proteomic profiles, and thereby genome rearrangements might contribute to strain variation and adaptation. To validate this assumption, we have performed genomic, transcriptomic, and proteomic analyses of recent clinical isolates from the Czech 
Republic obtained during 2008-2015, previously characterized vaccine strains isolated during 1954-1965 (22) (hereafter referred to as historic strains), and the reference strain Tohama I.

\section{Materials and Methods}

\section{Bacterial Strains and Growth Conditions}

Recent isolates of B. pertussis from the Czech Republic were obtained from the National Institute of Public Health in Prague (Table 1). Historic strains from the Czech Republic (22) and reference strain Tohama I (23) have been described. All strains were cultivated on Bordet-Gengou agar plates supplemented with $15 \%$ sheep blood for $3-4$ days at $37^{\circ} \mathrm{C}$. For liquid cultures, bacteria were grown in Stainer-Scholte medium (24) supplemented with $0.1 \%$ cyclodextrin and 0.5\% casamino acids (Difco, https://www.fishersci. com) at $37^{\circ} \mathrm{C}$. To harvest samples for DNA, RNA, and protein isolation, $B$. pertussis cells were grown overnight in Stainer-Scholte medium to mid-exponential phase of growth (optical density $\approx 1.0$ ). Three independent cultivations were performed to collect 3 biologic replicates of each of the strains for RNA and protein isolation.

\section{Genomic Analyses}

For the genome organization analysis, genomic sequences were aligned by using the progressive Mauve algorithm (25) and clustered on the basis of their genome organization similarity by using the maximum-likelihood for the gene order pipeline (26). For single-nucleotide polymorphism (SNP) analysis, IS elements within the genomes were masked with $\mathrm{Ns}$, and resulting sequences were aligned by using Mugsy software (27). SNPs were extracted by using custom scripts (https://genohub.com). Maximumparsimony phylogenetic analysis was performed on sequences with masked IS elements by using the kSNP3 program with a k number of 23 (28). The unrooted phylogenetic tree was visualized by using iTOL (29).

\section{RNA Isolation, Sequencing, and Data Analysis}

We provide information on RNA isolation, sequencing, and data analysis (Appendix 1, https:/ / wwwnc. cdc.gov/EID/article/27/1/19-1541-App1.pdf). RNA sequencing data from sequencing runs were deposited in the European Nucleotide Archive under project accession no. PRJEB34096. We defined significance as a $q$ value $<0.05$ ( $p$ value adjusted for multiple testing correction [Appendix 1]).

\section{Protein Sample Preparation and Proteomic Analysis}

We compiled information on protein sample preparation and label-free proteomic analysis, which used liquid chromatography with mass tandem spectrometry analyses (Appendix 1). Proteomics data were deposited in the ProteomeXchange Consortium by using the PRIDE partner repository with the dataset identifier PXD015184.

\section{Results}

\section{Genome Organization and Content of Recent Isolates}

We determined complete de novo genome assemblies of 9 recent isolates of $B$. pertussis strains collected in the Czech Republic during 2008-2015 from patients representing different age groups and vaccination status (Table 1). Genotyping of recent strains showed that they belonged to $p t x P 3$ lineage. SNP-based phylogenetic analysis of these strains and $>350$ complete $B$. pertussis genome sequences currently deposited in GenBank (Appendix 2 Table 1, https://wwwnc.cdc. gov/EID/article/27/1/19-1541-App2.xlsx) showed that recent $B$. pertussis isolates cluster with $p t x P 3$ isolates from other countries, demonstrating worldwide spread and lack of geographic signature (Figure 1). The genome alignment of recent isolates and previously characterized historic strains belonging to the ptxP1 lineage (22) showed that all genomes contain large-scale structural rearrangements (Figure 2, panel A). According to their genome organization, sequenced strains could be classified into 8 groups. None of the historic strains clustered with any of the

\begin{tabular}{|c|c|c|c|c|}
\hline \multirow[b]{2}{*}{ Year } & \multicolumn{2}{|c|}{ Strain information } & \multicolumn{2}{|c|}{ Patient information } \\
\hline & Name & Genotype & Age, y/sex & Vaccination status \\
\hline 2008 & Bp155 & ptxP3, fim2-1, fim 3B, prn2 & $<1 / \mathrm{M}$ & Not vaccinated \\
\hline 2008 & Bp312 & ptxP3, fim2-1, fim $3 B$, prn2 & $45 / F$ & $w P$ \\
\hline 2012 & Bp6260 & ptxP3, fim2-1, fim $3 A$, prn2 & $<1 / F$ & Not vaccinated \\
\hline 2012 & Bp6242 & ptxP3, fim2-1, fim $3 B$, prn2 & $67 / \mathrm{F}$ & Not vaccinated \\
\hline 2012 & Bp6384 & ptxP3, fim2-1, fim $3 A$, prn2 & 69/M & Not vaccinated \\
\hline 2012 & K10 & ptxP3, fim2-1, fim $3 B$, prn3 & $8 / F$ & $\mathrm{aP}$ \\
\hline 2014 & Bp82 & ptxP3, fim2-1, fim $3 A$, prn2 & $14 / F$ & wP plus aP \\
\hline 2014 & Bp46 & ptxP3, fim2-1, fim $3 A$, prn2 & $15 / \mathrm{M}$ & wP plus aP \\
\hline 2015 & Bp318 & ptxP3, fim2-1, fim $3 A$, prn2 & $7 / F$ & $\mathrm{aP}$ \\
\hline
\end{tabular}




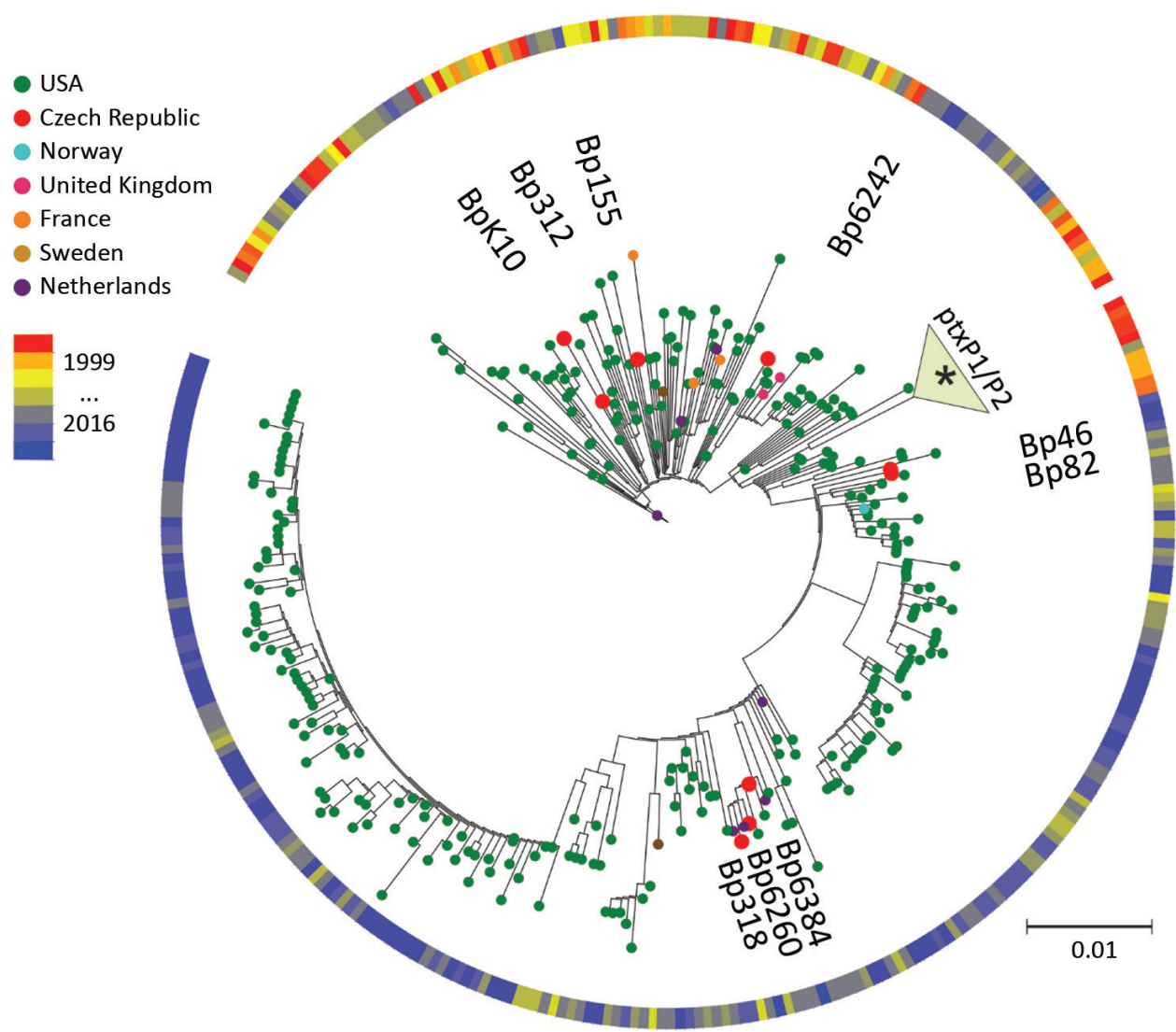

Figure 1. Maximumparsimony, unrooted phylogenetic tree based on single-nucleotide polymorphism analysis of available genome sequences of Bordetella pertussis. Red dots indicate recent isolates from the Czech Republic. Year and country of isolation are color-coded. The 3 black dots indicate time span between 1999 and 2016. Asterisk (*) indicates association of historic strains from the Czech Republic with the $p t x P 1 / p t x P 2$ clade. Scale bar indicates nucleotide substitutions per site. recent isolates. The separation of these 2 groups was verified by using a maximum-likelihood phylogenetic tree, which was constructed on the basis of the genome organization of all sequenced strains from the Czech Republic (Figure 2, panel B).

To check whether there are also sequence signatures differentiating these 2 groups of strains, we performed SNP analysis, which yielded 35 SNPs (15 synonymous, 13 nonsynonymous, and 7 intergenic) (Appendix 2 Table 2) and distinguished historic and recent isolates. Variants found in historic strains were also present in Tohama I. One of the new SNPs specific for recent isolates was identified in the promoter region of the bteA gene, which encodes the type III secretion system (T3SS) effector. Approximately one third of the SNPs have been reported to be specific for the $p t x P 3$ lineage isolates from other countries (30). When compared with those of historic strains, the genome size of recent isolates was substantially reduced, thereby confirming ongoing gene loss within the global population of B. pertussis (Appendix 2 Table 3). Analysis of genome alignments showed 2 regions of difference (RD) between the 2 groups of strains from the Czech Republic, which corresponded to previously identified regions RD3 and RD10 (20). Although RD3 (28.7 kb, spans genes BP0910A-BP0937) is absent in all recent isolates, the RD10 $(25.1 \mathrm{~kb}$, spans genes BP1948-BP1968) is absent in all recent isolates and historic strain V67.

\section{Transcriptomic Profiles and Genomic Structure Alterations}

Total RNA was isolated from biologic triplicates of B. pertussis Tohama I strain; historic strains VS393, VS67, and VS401; and recent isolates Bp318, Bp155, Bp46, Bp6242, and BpK10 and analyzed by using RNA sequencing. These strains were selected on the basis of genome organization and phylogenetic distances to encompass the highest variability among the studied strains (Figure 2, panel B). Hierarchical clustering of RNA sequence data showed that samples from both groups of strains from the Czech Republic clustered separately from each other and from Tohama I (Figure 3). Consistent with phylogenetic analysis (Figure 2, panel B), we found that samples of strain VS67 formed a separate cluster. These analyses suggested that among historic strains, the VS67 strain displays closest distance to recent strains, which is consistent with our 
previous observation that the VS67 strain clusters together with a recent U.S. isolate (22).

Differential expression (DE) analysis identified 78,124 , and 115 significantly ( $q<0.05$ for all comparisons) modulated $B$. pertussis genes $\left(-1 \geq \log _{2} F C \geq 1\right)$ between recent isolates and historic strains, recent isolates and Tohama I, and historic strains and Tohama I, respectively (Appendix 2 Table 4). Among the DE genes, 30 were up-regulated in recent isolates compared with historic strains, including those encoding the flagella apparatus (flgB- $)$ ), LuxR (BP1969), and ArsR (BP2946) families of transcriptional regulators, phosphoglucomutase ( $\mathrm{pgm})$, phosphoglucose isomerase (pgi), and nicotinate-nucleotide diphosphorylase (nadC). Conversely, among the $48 \mathrm{DE}$ genes down-regulated in recent isolates were genes encoding the polysaccharide capsule proteins ( $k p s E M T)$, several ABC transporters, and central metabolism enzymes, including those involved in tryptophan synthesis $(\operatorname{trpDEG})$. Expression of several virulence

A

VS366, VS377, VS401
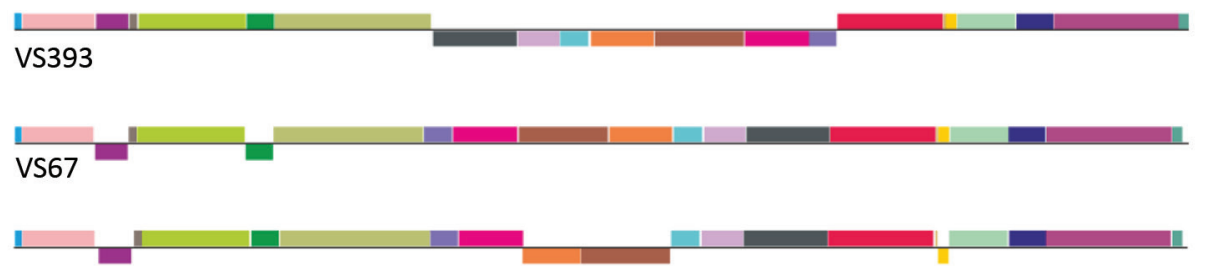

Bp6384, Вp6260, Вp318

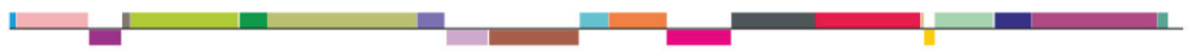

Bp82, Bp46

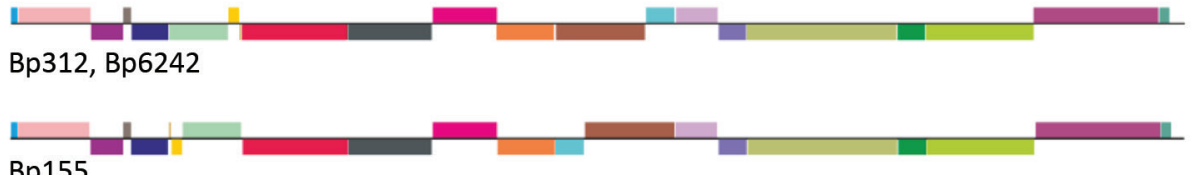

Bp155

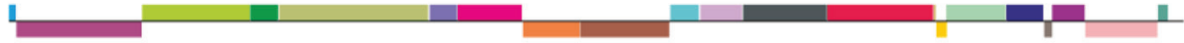

BpK10

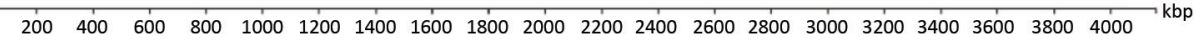
Nucleotide position
Figure 2. Genomic analyses of Bordetella pertussis isolates from the Czech Republic. A) Genome alignment of historic and recent isolates showing largescale genome rearrangements. Homologous gene blocks are denoted by the same color. B) Maximum-likelihood phylogenetic tree based on genomic organization of historic (blue) and recent (red) isolates. Asterisk $\left({ }^{*}\right)$ indicates strains selected for transcriptomic and proteomic analyses. Scale bar indicates nucleotide substitutions per site. kbp, kilobasepairs.

\section{B}

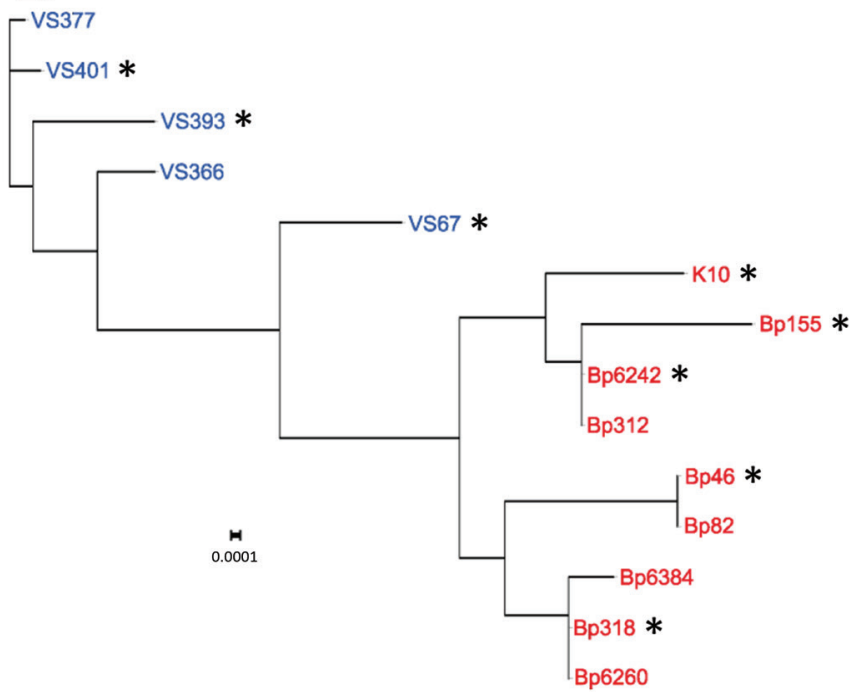




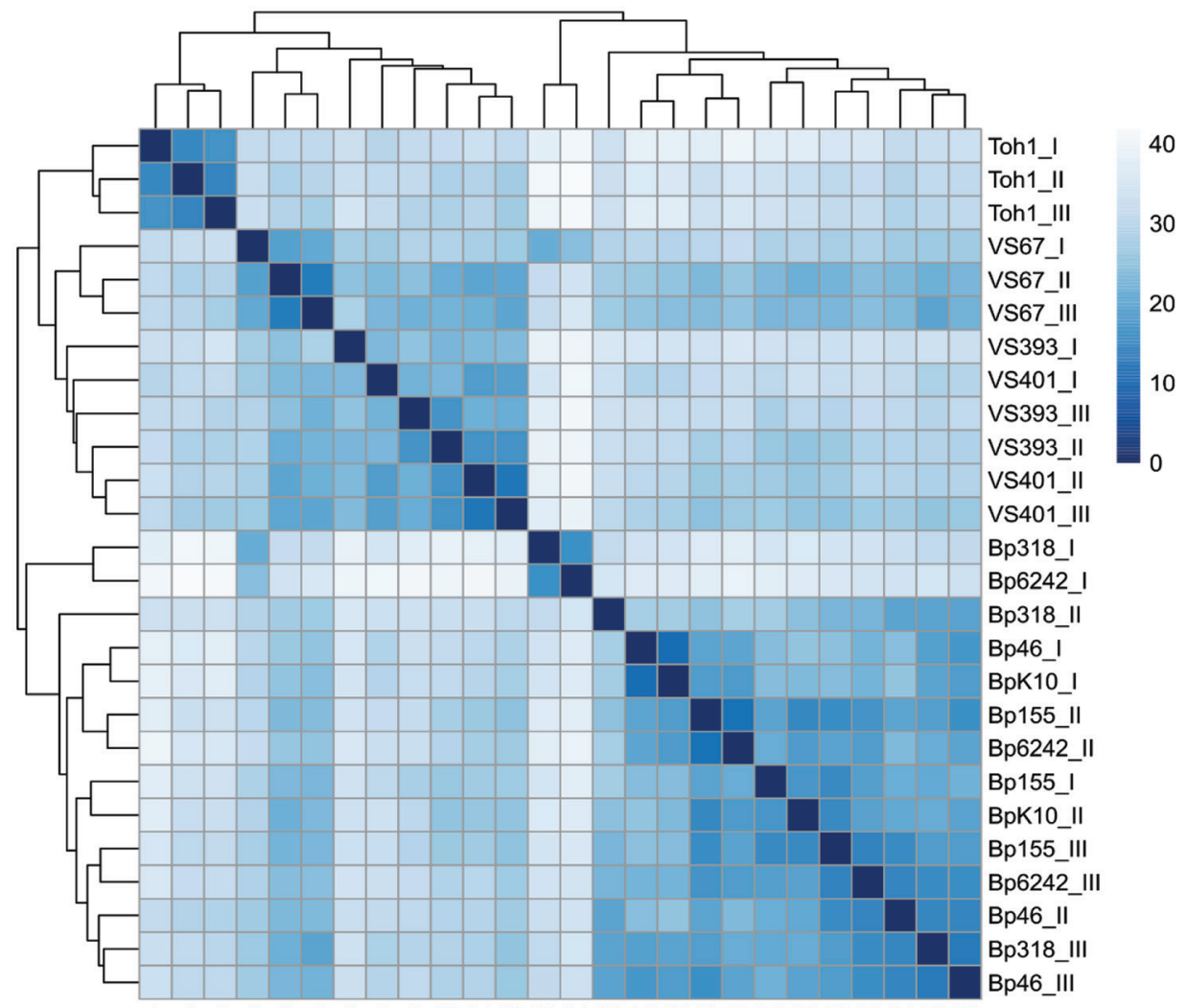

Figure 3. Heat map of sampleto-sample distances showing the hierarchical clustering of historic and recent isolates of Bordetella pertussis from the Czech Republic and the Tohama I strain. Colored scale bar and numbers on the right indicate Euclidian distances between samples calculated by using rlog-normalized RNA sequence data. 1, Toh1_1; 2 , Toh1_II; 3, Toh1_III; 4, VS67_1; 5, VS67_II; 6, VS67_III; 7, VS93_I; 8,VS401_I; 9,VS393_III; 10, VS393_II; 11, VS401_II; 12,VS401_III; 13, Bp318_I; 14, Bp6242_I; 15, Bp318_II; 16, Bp46 ।; 17, BpK10 ।; 18, Bp155_II; 19, Bp6242_II; 20, Bp155_I; 21, BpK10_II; 22, Bp155_III; 23, Bp6242_III; 24, Bp46_II; 25, Bp318_III; 26, Bp46_III.

12234456678891011121314151617181920212223242526

factors, including pertactin, tracheal colonization factor, filamentous hemagglutinin, and pertussis toxin subunit S3, was significantly up-regulated in recent isolates. However, the increase did not reach the 2-fold threshold. A recent isolate-specific SNP, which was identified in the promoter region of the $b t e A$ gene, did not result in a significant change of gene expression (Appendix 2 Table 4). Among the DE genes that showed increased expression in both groups of strains from the Czech Republic compared with Tohama I, we identified numerous genes within the T3SS bcs/btr locus, including bsp22, bopN, bop $B$, and bopD and several genes involved in sulfate metabolism (cysADITW).

Gene ontology enrichment (Figure 4, panel A) showed that within the set of genes, which were significantly modulated between recent and historic isolates, categories such as bacterial type flagellumdependent cell motility, polysaccharide biosynthesis, and tryptophan biosynthesis were highly enriched. Conversely, when we compared both groups of isolates from the Czech Republic to Tohama I (Figure 4, panel C), genes associated with sulfate transmembrane transport, pathogenesis, and protein secretion by the type III secretion system terms were enriched among the DE genes.

\section{Clustering of Proteomic Profiles of Recent Isolates,} Historic Strains, and Tohama I Strain

The cell-associated (bacterial pellets) and cell-free (culture supernatants) fractions of selected B. pertussis strain cultures were analyzed by using liquid chromatography with mass tandem mass spectrometry. First, hierarchical clustering of the cell-associated protein profiles showed that consistent with RNA sequencing data, strains from the Czech Republic cluster separately from Tohama I and despite high variability among biologic replicates, separation of historic and recent strains was still apparent (Figure 5, panel A). Similarly, hierarchical clustering of secreted proteins indicated that recent isolates cluster apart from historic strains and Tohama I (Figure 5, panel B).

Label-free quantification of cell-associated protein intensities identified 33, 132, and 87 proteins showing significantly changed abundance between recent and historic strains, recent strains and Tohama I, and historic strains and Tohama I, respectively (Appendix 2 Table 5). In good correlation with transcriptomic data, we found that protein levels of hydroxymethyglutaryl-CoA lyase (BP3695), ArsR family transcriptional factor BP2946, small lipoprotein BP2782, and nicotinate-nucleotide diphosphorylase NadC were increased, but levels of several central metabolism 
enzymes (BP0624-BP0629), tryptophan synthesis genes, and polysaccharide capsule proteins were decreased in recent isolates compared with historic strains (Table 2). Also in support of RNA sequencing data, we determined that various components of the T3SS apparatus and several proteins involved in metabolism of sulfate showed increased abundance compared with Tohama I (Table 3). All strains from the Czech Republic produced pertactin, but flagellar proteins were not detected in any of the studied strains.

Label-free quantification analysis of secreted proteins showed that 121,130 , and 43 proteins displayed significant changes in abundance between recent and historic strains, recent strains and Tohama I, and historic strains and Tohama I, respectively (Appendix 2 Table 6). Levels of several secreted proteins were in good agreement with transcriptomic data (e.g., strains from the Czech Republic and in particular recent isolates secreted increased amounts of several T3SS proteins compared with Tohama I) (Table 3). Abundance of all pertussis toxin subunits and associated transport protein PtlE was higher in recent isolates than in historic strains, which suggests that some of the differences between the $p \operatorname{txP} 1$ and $p \operatorname{txp} 3$ strains are also manifested at the level of protein secretion.

\section{Changes in Genome Structure and Alterations in Gene Expression}

Considering the observed differences between historic and recent strains, we attempted to track back the modulated gene expression profiles to alterations in the genome sequence and structure. Because our SNP analysis (Table 2) suggested that there were no SNPs that could explain the altered expression of the DE genes, we have additionally inspected the upstream regions of all DE genes for larger sequence variations. We identified such variations in 4 genes. The gene toh_02779 (BP2827) was preceded by an IS481 element in Bp155, Bp6242, and BpK10, but not in other strains. In addition, $3 \mathrm{DE}$ genes with an upstream IS481 element had varying gene context further upstream of the transposase in the studied strains (Table 4). Apparently, the observed differences in expression of these genes could be potentially linked to the upstream IS elements.
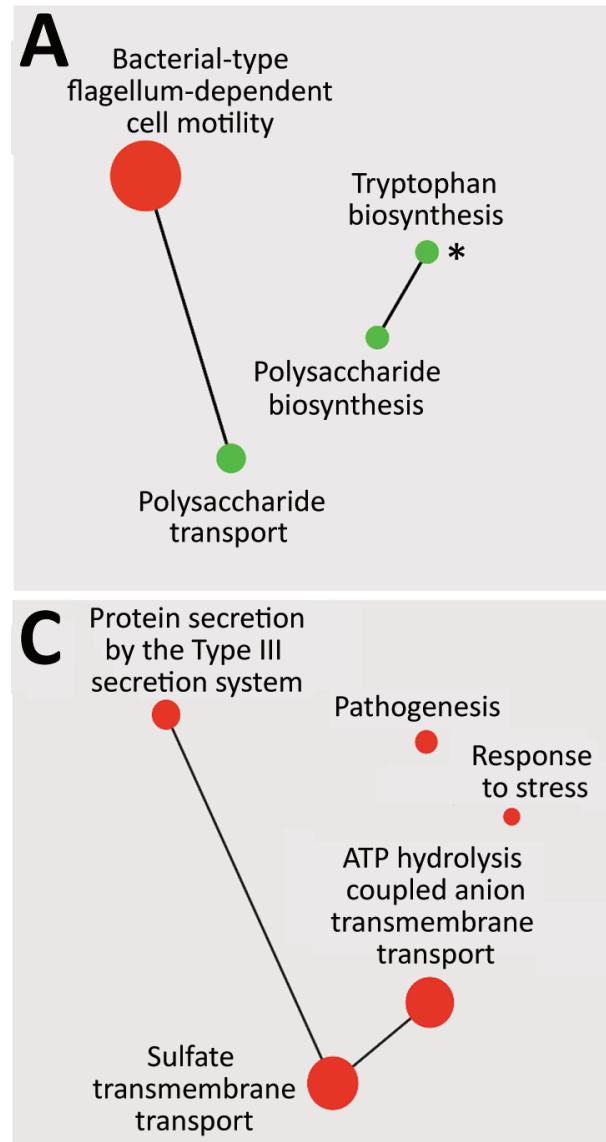

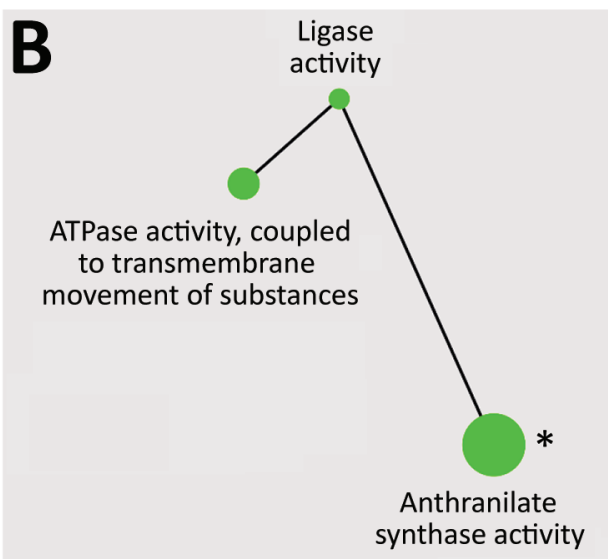

D

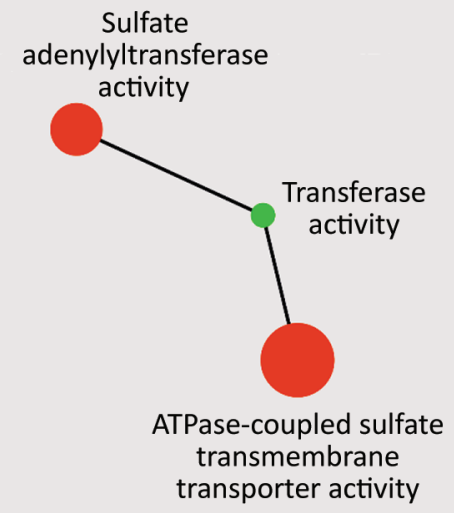

Figure 4. Gene ontology enrichment analysis of genes down-regulated or up-regulated between recent and historic strains of Bordetella pertussis from the Czech Republic (A, $B)$ or between both groups of strains from the Czech Republic and Tohama I (C, D). Enriched terms from the domains' biological process $(A, C)$ and molecular function $(B, D)$ and their catenations, shown as green circles (downregulated genes) and red circles (up-regulated genes), were summarized by using Revigo (http://revigo.irb. hr) and visualized by using Cytoscape (https://cytoscape. org) as interactive scatter plots. Circle size indicates level of enrichment. Asterisks $\left(^{*}\right)$ in panels $A$ and $B$ indicate gene ontology terms that were enriched also for genes downregulated in recent isolates compared with Tohama I. 

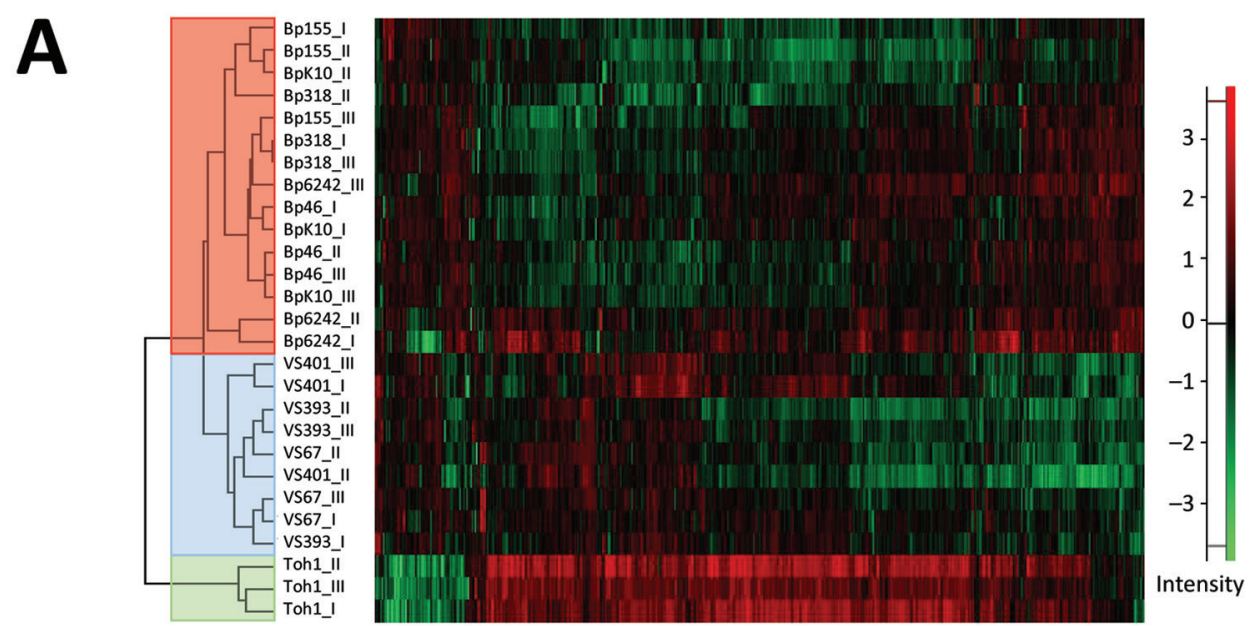

Figure 5. Heatmaps showing hierarchical clustering performed on Z-score normalized $\log _{2}-$ transformed label-free intensity values of cell-associated $(A)$ or secreted $(B)$ protein fractions of historic and recent isolates of Bordetella pertussis from the Czech Republic and the Tohama I strain. Clustering of recent, historic, and Tohama I strains is indicated by red, blue, and green, respectively. Scale bars indicate intensity of proteins normalized by Z-score.
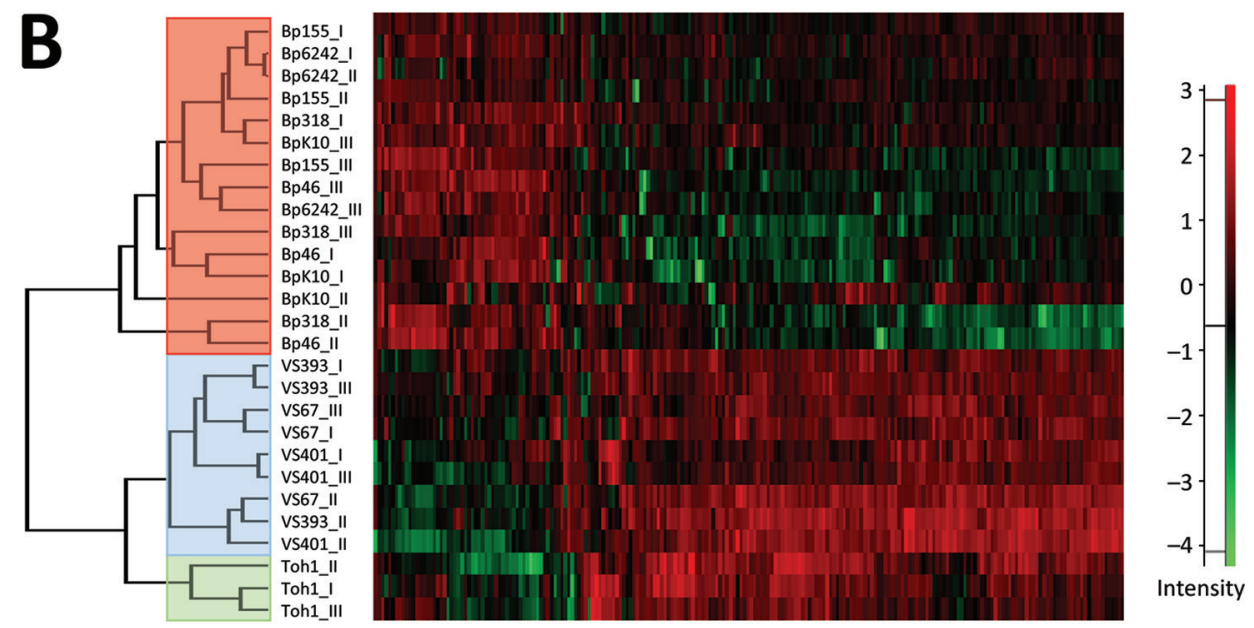

We then tested the possible effect of genome rearrangements on the distance of the DE genes from the origin of replication (oriC), a parameter that can greatly affect gene expression (31). We determined the distance from oriC to all the genes significantly deregulated between recent and historic strains, and although expression of some of the genes inversely correlated with the distance from oriC, the differences were not significant.

\section{Discussion}

We conducted a comparative study analyzing the link between genomic organization, gene expression profiles, and protein production/secretion in historic and recent strains of $B$. pertussis. Our results indicate that global changes in genomic structures observed between historic and recent isolates of B. pertussis from the Czech Republic translated into different gene expression and protein production profiles. Similarly to other countries, the IS element-driven recombination led to large changes in genomic structures and to considerable gene loss in the isolates from the Czech Republic over the past 50-60 years. Results of our integrated omics analysis support our assumption that genomic rearrangements might affect global expression profiles and phenotypic diversity in B. pertussis. Hierarchical clustering of our omics data indicates that strains, which cluster apart at genomic structure level, also have distinct transcriptomic and proteomic profiles.

Given the extent of genome structural variability among both groups of strains, the number of differentially expressed genes was rather low $(\approx 2 \%$ of all coding genes). Earlier DNA microarray studies suggested that gene expression profiles between $p t x P 1$ strains and recent resurgence-associated $p t x P 3$ lineage differ only subtly $(32,33)$. Although we have identified an increased number of significantly modulated genes, our data on historic ( $p t x P 1)$ and recent ( $p t x P 3)$ isolates are consistent with these reports. None of the gene expression alterations could be shown to result from nucleotide polymorphism, and only a few could be 
Table 2. Genes expressing consistently changed RNA and protein levels between recent and historic isolates of Bordetella pertussis, Czech Republic*

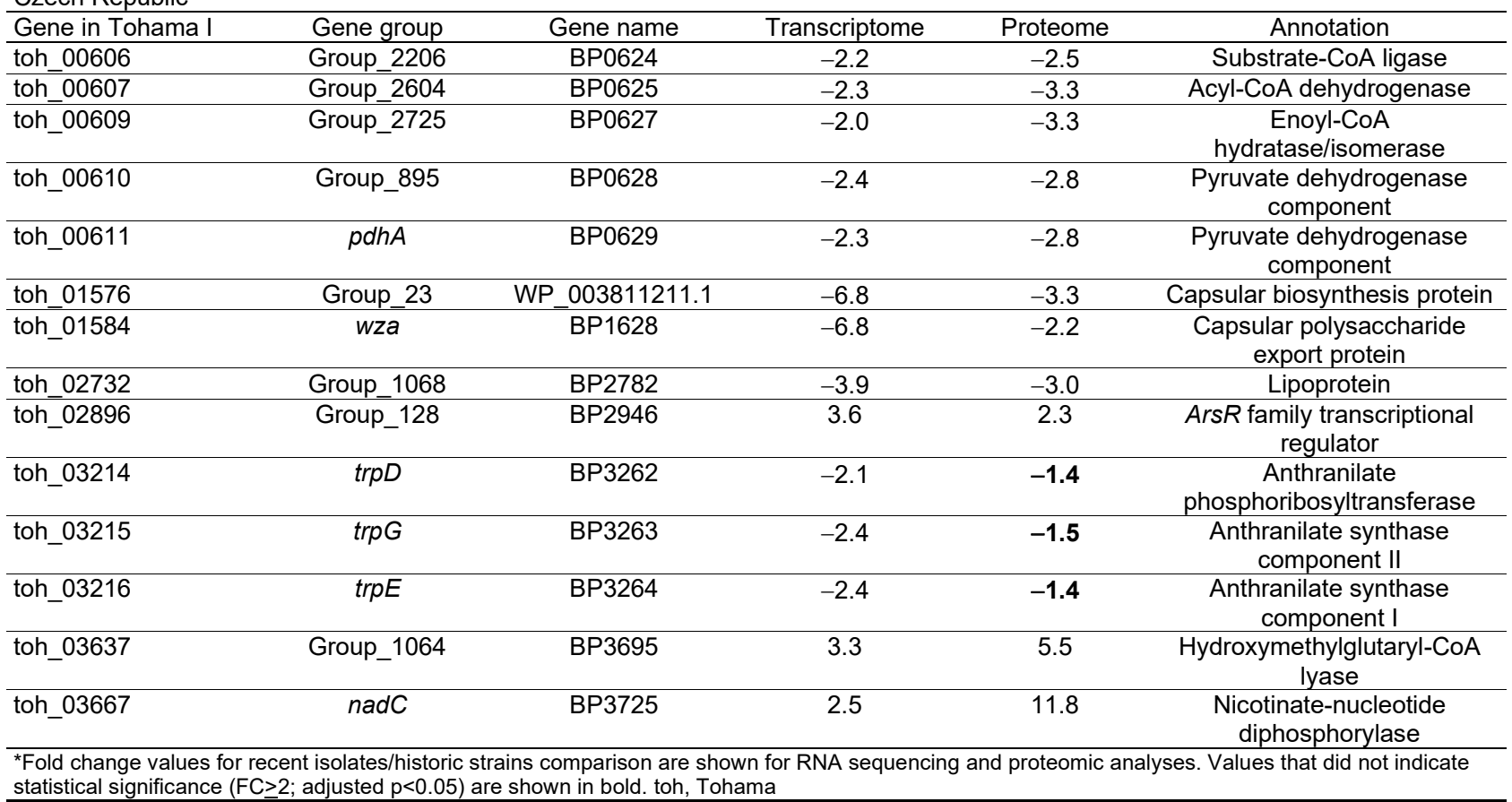

linked to IS element-induced changes in the local gene context. Upstream of the IS481 element adjacent to the BP1492 gene, we identified the BPt20 tRNA gene in most historic strains and Tohama I, which is, however, missing in recent isolates and historic strain VS67. Thus, it is possible that the activity of the strong tRNA promoter is responsible for increased expression of the BP1492 gene in historic strains. Also, the presence of an IS481 element in front of the BP2827 gene in Bp155, Bp6242, and BpK10 strains might explain the increased expression of this gene in recent isolates. In support of this possibility, when compared with all other strains lacking this IS element, these 3 recent isolates showed highly increased expression of this gene. It is also possible that the observed differences in gene expression between historic and recent strains result from changes in genome organization or gene loss. Bacterial chromosome organization appears to favor a conserved gene order (34), and changes in genome architecture and topology can affect gene expression $(35,36)$. Therefore, it is conceivable that genome rearrangements, resulting in changes in gene order and orientation or in large deletions, might affect transcriptomic profiles in B. pertussis.

We have identified 2 previously characterized regions of difference between historic and recent strains, which might offer alternative explanation for the observed differences in gene expression $(20,37)$. Consistent with these reports, our reports found that genes within RD3 and RD10 are missing in all recent isolates. RD3 contains 2 putative transcriptional regulators (BP0924 and BP0928) of unknown function. Thus, it is probable that absence of these regulators in recent isolates might be accountable for some of the identified alterations in gene expression.

Furthermore, RNA sequencing analysis identified 2 transcriptional regulator genes that are expressed at higher levels in recent isolates, and suggested that some of the observed differences between historic and recent strains might also result from altered expression of regulatory genes. BP1969, which encodes a LuxR family transcriptional factor, lies upstream of the BP1970 and BP1971 genes, which encode phosphoglucomutase Pgm and phosphoglucose isomerase Pgi. Similarly to BP1969, pgm and pgi genes were significantly up-regulated in recent strains. Therefore, we assume that the BP1969 gene probably represents a cognate regulator for these glycolytic genes. Besides its role in glycolysis, Pgm catalyzes the generation of sugar nucleotides needed for biosynthesis of lipopolysaccharide and cell wall and was shown to be required for virulence of B. bronchiseptica (38) and several other pathogens $(39,40)$. Strains lacking the $\mathrm{pgm}$ gene showed increased susceptibility to antimicrobial peptides and were attenuated in in vivo models of infection (38-40). Pgi catalyzes the second step in glycolysis and was shown to be required for virulence of Xanthomonas campestris (41). Thus, we presume that 
Table 3. Genes expressing consistently increased RNA and protein levels in historic and recent isolates of Bordetella pertussis, Czech Republic, compared with Tohama I*

\begin{tabular}{|c|c|c|c|c|c|c|c|c|c|}
\hline \multirow{2}{*}{$\begin{array}{l}\text { Gene in } \\
\text { Tohama I }\end{array}$} & \multirow[b]{2}{*}{ Gene group } & \multirow[b]{2}{*}{ Gene name } & \multicolumn{2}{|c|}{ Transcriptome } & \multicolumn{2}{|c|}{ Proteome } & \multicolumn{2}{|c|}{ Secretome } & \multirow[b]{2}{*}{ Annotation } \\
\hline & & & Cz/Toh & VS/Toh & Cz/Toh & VS/Toh & Cz/Toh & VS/Toh & \\
\hline toh 00485 & Group 1902 & BP0500 & 2.1 & 2.8 & 1.5 & 1.3 & 6.0 & 5.1 & T3SS effector BopC \\
\hline toh_00936 & $S \overline{b p}$ & BP0966 & 14.7 & 13.0 & 5.6 & 4.6 & ND & ND & Sulfate-binding protein \\
\hline toh 02200 & bscl & BP2249 & 2.1 & 3.1 & 1.2 & ND & 2.8 & 6.2 & T3SS protein Bscl \\
\hline toh_02203 & bopB & BP2252 & 2.1 & 3.0 & 3.1 & 2.8 & 2.1 & 1.8 & T3SS protein BopB \\
\hline toh_02204 & bopD & BP2253 & 1.8 & 2.5 & 2.0 & 1.5 & 3.7 & 2.3 & T3SS protein BopD \\
\hline toh_02205 & bcrH1 & BP2254 & 2.3 & 4.0 & 2.5 & 2.1 & 1.6 & 3.1 & T3SS protein \\
\hline toh 02206 & Group 1710 & BP2255 & 2.4 & 3.8 & 3.9 & 4.0 & 4.8 & 1.7 & Hypothetical protein \\
\hline toh 02207 & bsp22 & BP2256 & 2.4 & 3.6 & 1.6 & 1.5 & 3.6 & 1.8 & T3SS protein Bsp22 \\
\hline toh 02208 & bopN & BP2257 & 2.5 & 2.9 & 1.3 & 1.0 & 3.1 & 1.5 & T3SS protein BopN \\
\hline toh 02210 & Group 2630 & BP2259 & 2.0 & 2.4 & 1.4 & 1.9 & 5.1 & 2.4 & Putative T3SS protein \\
\hline toh 02214 & $b s c \bar{c}$ & BP2263 & 2.3 & 1.5 & 5.2 & 4.5 & 2.0 & 2.3 & T3SS protein BscE \\
\hline toh_03375 & Group_1130 & BP3434 & 6.1 & 4.4 & 3.3 & 1.9 & 2.6 & 1.8 & Exported protein \\
\hline
\end{tabular}

increased expression and production of both enzymes might contribute to increased virulence and fitness of the $p$ txP3 lineage.

Among other modulated genes, expression of numerous genes within the operon encoding the flagellar apparatus was significantly increased in recent isolates. However, we could not corroborate this finding because we did not detect any flagellar proteins in our samples. Recent observations suggest that $B$. pertussis is motile under modulatory Bvg-conditions (42) and that motility genes are up-regulated during adaptation to the mouse respiratory tract (43). Apparently, in vivo conditions, prevailing during $B$. pertussis infections in mice, cannot be completely reproduced under standard laboratory growth conditions, as documented $(43,44)$, and further experiments are required to determine whether the increased expression translates into higher motility of recent isolates and contributes to improved ability of $p t x P 3$ strains to colonize the respiratory tract (33).

Conversely, expression of an almost complete operon that encodes genes involved in polysaccharide capsule synthesis was substantially downregulated in recent isolates. This observation is consistent with that of a previous report (45) and demonstrates that capsule proteins are produced by $B$. pertussis. This finding also involves the protein responsible for polysaccharide biosynthesis TviD (BP1618), which has been reported to be encoded by a pseudogene (14).

Data on the role of the capsule in the virulence and physiologic fitness of $B$. pertussis are contradictory. Hoo et al. (46) showed that the capsule proteins are expressed during the infection and are required for an efficient colonization of mouse lungs. In contrast, in vitro assays showed that the capsule did

Table 4. Proteins encoded in regions upstream of an IS481 element adjacent to differentially expressed genes in recent and historic strains of Bordetella pertussis, Czech Republic*

\begin{tabular}{|c|c|c|c|}
\hline \multirow[b]{2}{*}{ Strain } & \multicolumn{3}{|c|}{ Protein } \\
\hline & toh_01451 (BP1492) & toh_01915 (BP1969) & toh_02005 (BP2055) \\
\hline Tohama I & tRNA & $\begin{array}{l}\text { Partial phosphonate } \\
\text { monoester hydrolase }\end{array}$ & Partial cyclopropane-fatty-acyl-phospholipid synthase \\
\hline VS393 & tRNA & $\begin{array}{l}\text { Partial phosphonate } \\
\text { monoester hydrolase }\end{array}$ & Partial cyclopropane-fatty-acyl-phospholipid synthase \\
\hline VS401 & tRNA & $\begin{array}{l}\text { Partial phosphonate } \\
\text { monoester hydrolase }\end{array}$ & IS481 element \\
\hline VS67 & Partial FUSC family protein & $\begin{array}{l}\text { Partial phosphonate } \\
\text { monoester hydrolase }\end{array}$ & Partial cyclopropane-fatty-acyl-phospholipid synthase \\
\hline Bp318 & Partial FUSC family protein & $\begin{array}{l}\text { MarR family transcriptional } \\
\text { regulator }\end{array}$ & Partial cyclopropane-fatty-acyl-phospholipid synthase \\
\hline Bp155 & Partial FUSC family protein & $\begin{array}{l}\text { MarR family transcriptional } \\
\text { regulator }\end{array}$ & Partial cyclopropane-fatty-acyl-phospholipid synthase \\
\hline Bp46 & Partial FUSC family protein & $\begin{array}{l}\text { MarR family transcriptional } \\
\text { regulator }\end{array}$ & Partial cyclopropane-fatty-acyl-phospholipid synthase \\
\hline Bp6242 & Partial FUSC family protein & $\begin{array}{l}\text { MarR family transcriptional } \\
\text { regulator }\end{array}$ & Partial cyclopropane-fatty-acyl-phospholipid synthase \\
\hline BpK10 & Partial FUSC family protein & $\begin{array}{l}\text { MarR family transcriptional } \\
\text { regulator }\end{array}$ & Partial cyclopropane-fatty-acyl-phospholipid synthase \\
\hline
\end{tabular}


not protect $B$. pertussis cells from phagocytosis and serum killing (45) and that the capsule locus was not expressed during infection of mouse respiratory tract (43). Therefore, it is difficult to assess whether reduced production of capsule proteins provides recent strains with any selective advantage. Nevertheless, the capsular polysaccharides of several gramnegative bacteria are highly immunogenic and were used to formulate carbohydrate-protein conjugate vaccines (47). Therefore, it is possible that in circulating isolates of $B$. pertussis, reduced production of the capsule synthesis apparatus contributes to evasion from the host immune response.

Our omics data manifest that, in spite of being isolated at the similar period of time, historic strains are substantially distinct from the reference strain Tohama I. Previous genomic analyses documented that several different clusters of $B$. pertussis circulated in Europe and the United States already in prevaccine and early vaccine eras and that their genomes were different from Tohama I (20,37). Our results with strains from the Czech Republic are consistent with these observations and also confirm this distinction at the transcriptomic and proteomic levels. For example, expression and production of various sulfate metabolism factors (sbp, cys $T, c y s A)$ were strongly reduced in Tohama I compared with strains from the Czech Republic. Likewise, we demonstrated that recent and historic strains had significantly increased expression, production, and secretion of several T3SS components. This observation is consistent with previous reports $(48,49)$ and confirms that not only recent isolates but also low-passage historic strains of B. pertussis are T3SS proficient $(48,49)$. We conclude that, in agreement with previous reports $(37,50)$, the Tohama I strain is not a good representative of the circulating B. pertussis population.

Collectively, our data suggest that, besides shaping the evolution of $B$. pertussis on a genomic scale, the genome rearrangement and genome reduction processes also affect global transcriptomic and proteomic profiles. In agreement with results of a previous report (20), we assume that these mechanisms counterbalance the low level of genetic variability observed in this pathogen and strongly contribute to adaptation of the global population of B. pertussis.

\section{Acknowledgments}

We thank Richard Neuboeck helping to setup software for some of the analyses, Michael Weigand for providing the sequence of the oriC region, and Karel Harant and Pavel Talacko for performing the liquid chromatography with mass tandem spectrometry analyses runs.
This study was supported by Czech Health Research Council (http:/ /www.azvcr.cz) grant 16-30782A to B.V., Czech Research Foundation (http:/ / www.qacr.cz) grant 19-12338S to B.V., institutional funding RVO61388971, and a mobility grant from the Czech Academy of Sciences (MSM200201702) to A.D. This study was also supported by the project "BIOCEV - Biotechnology and Biomedicine Centre of the Academy of Sciences and Charles University" (CZ.1.05/1.1.00/02.0109) from the European Regional Development Fund.

\section{About the Author}

Dr. Dienstbier is a postdoctoral fellow in the Laboratory of Post-transcriptional Control of Gene Expression, Institute of Microbiology, Czech Academy of Sciences, Prague, Czech Republic. Her research interests are genomics and transcriptomics of $B$. pertussis strains and molecular mechanisms underlying the pathogenesis of pertussis.

\section{References}

1. Centers for Disease Control and Prevention. Vaccine preventable deaths and the Global Immunization Vision and Strategy, 2006-2015. MMWR Morb Mortal Wkly Rep. 2006;55:511-5.

2. Raguckas SE, VandenBussche HL, Jacobs C, Klepser ME. Pertussis resurgence: diagnosis, treatment, prevention, and beyond. Pharmacotherapy. 2007;27:41-52. https:/ / doi.org/ 10.1592/phco.27.1.41

3. van Gent M, Heuvelman CJ, van der Heide HG, Hallander HO, Advani A, Guiso N, et al. Analysis of Bordetella pertussis clinical isolates circulating in European countries during the period 1998-2012. Eur J Clin Microbiol Infect Dis. 2015;34:821-30. https://doi.org/10.1007/s10096-014-2297-2

4. Fabiánová K, Benes C, Kríz B. A steady rise in incidence of pertussis since nineties in the Czech Republic. Epidemiol Mikrobiol Imunol. 2010;59:25-33.

5. Sealey KL, Belcher T, Preston A. Bordetella pertussis epidemiology and evolution in the light of pertussis resurgence. Infect Genet Evol. 2016;40:136-43. https://doi.org/10.1016/j.meegid.2016.02.032

6. Esposito S, Stefanelli P, Fry NK, Fedele G, He Q, Paterson P, et al.; World Association of Infectious Diseases and Immunological Disorders (WAidid) and the Vaccine Study Group of the European Society of Clinical Microbiology and Infectious Diseases (EVASG). Pertussis prevention: reasons for resurgence, and differences in the current acellular pertussis vaccines. Front Immunol. 2019;10:1344. https://doi.org/10.3389/fimmu.2019.01344

7. Cherry JD. Epidemic pertussis and acellular pertussis vaccine failure in the 21st century. Pediatrics. 2015;135:11302. https://doi.org/10.1542/peds.2014-4118

8. Klein NP, Bartlett J, Rowhani-Rahbar A, Fireman B, Baxter R. Waning protection after fifth dose of acellular pertussis vaccine in children. N Engl J Med. 2012;367:1012-9. https://doi.org/10.1056/NEJMoa1200850

9. Liko J, Robison SG, Cieslak PR. Priming with whole-cell versus acellular pertussis vaccine. N Engl J Med. 2013;368:581-2. https://doi.org/10.1056/NEJMc1212006

10. Burdin N, Handy LK, Plotkin SA. What is wrong with pertussis Vvccine immunity? The problem of waning effectiveness of 
pertussis vaccines. Cold Spring Harb Perspect Biol. 2017;9:a029454. https://doi.org/10.1101/cshperspect.a029454

11. Bart MJ, Harris SR, Advani A, Arakawa Y, Bottero D, Bouchez V, et al. Global population structure and evolution of Bordetella pertussis and their relationship with vaccination. MBiol. 2014;5:e01074. https:// doi.org/10.1128/mBio.01074-14

12. Mooi FR, Van Der Maas NA, De Melker HE. Pertussis resurgence: waning immunity and pathogen adaptation: two sides of the same coin. Epidemiol Infect. 2014;142:685-94. https://doi.org/10.1017/S0950268813000071

13. Bouchez V, Hegerle N, Strati F, Njamkepo E, Guiso N. New data on vaccine antigen deficient Bordetella pertussis isolates. Vaccines (Basel). 2015;3:751-70. https://doi.org/10.3390/ vaccines 3030751

14. Parkhill J, Sebaihia M, Preston A, Murphy LD, Thomson N, Harris DE, et al. Comparative analysis of the genome sequences of Bordetella pertussis, Bordetella parapertussis and Bordetella bronchiseptica. Nat Genet. 2003;35:32-40. https://doi.org/10.1038/ng1227

15. Cummings CA, Brinig MM, Lepp PW, van de Pas S, Relman DA. Bordetella species are distinguished by patterns of substantial gene loss and host adaptation. J Bacteriol. 2004;186:1484-92. https:// doi.org/10.1128/JB.186.5.14841492.2004

16. Linz B, Ivanov YV, Preston A, Brinkac L, Parkhill J, Kim M, et al. Acquisition and loss of virulence-associated factors during genome evolution and speciation in three clades of Bordetella species. BMC Genomics. 2016;17:767. https:/ / doi.org/10.1186/s12864-016-3112-5

17. Bowden KE, Weigand MR, Peng Y, Cassiday PK, Sammons S, Knipe K, et al. Genome structural diversity among 31 Bordetella pertussis isolates from two recent U.S. whooping cough statewide epidemics. MSphere. 2016; 1:e00036-16. https:// doi.org/10.1128/mSphere.00036-16

18. Weigand MR, Peng Y, Loparev V, Batra D, Bowden KE, Burroughs $\mathrm{M}$, et al. The history of Bordetella pertussis genome evolution includes structural rearrangement. J Bacteriol. 2017;199:e00806-16. https:/ / doi.org/10.1128/ JB.00806-16

19. Ring N, Abrahams JS, Jain M, Olsen H, Preston A, Bagby S. Resolving the complex Bordetella pertussis genome using barcoded nanopore sequencing. Microb Genom. 2018;4. https:/ / doi.org/10.1099/mgen.0.000234

20. Brinig MM, Cummings CA, Sanden GN, Stefanelli P, Lawrence A, Relman DA. Significant gene order and expression differences in Bordetella pertussis despite limited gene content variation. J Bacteriol. 2006;188:2375-82. https:/ / doi.org/10.1128/JB.188.7.2375-2382.2006

21. Amman F, D'Halluin A, Antoine R, Huot L, Bibova I, Keidel K, et al. Primary transcriptome analysis reveals importance of IS elements for the shaping of the transcriptional landscape of Bordetella pertussis. RNA Biol. 2018; 15:967-75. https://doi.org/10.1080/15476286.2018.1462655

22. Dienstbier A, Pouchnik D, Wildung M, Amman F, Hofacker IL, Parkhill J, et al. Comparative genomics of Czech vaccine strains of Bordetella pertussis. Pathog Dis. 2018;76. https://doi.org/10.1093/femspd/fty071

23. Kasuga T, Nakase Y, Ukishima K, Takatsu K. Studies on Haemophilis pertussis. III. Some properties of each phase of H. pertussis. Kitasato Arch Exp Med. 1954;27:37-47.

24. Stainer DW, Scholte MJ. A simple chemically defined medium for the production of phase I Bordetella pertussis. J Gen Microbiol. 1970;63:211-20. https://doi.org/10.1099/ 00221287-63-2-211

25. Darling AE, Mau B, Perna NT. progressiveMauve: multiple genome alignment with gene gain, loss and rearrangement.
PLoS One. 2010;5:e11147. https:/ / doi.org/10.1371/ journal.pone.0011147

26. Hu F, Lin Y, Tang J. MLGO: phylogeny reconstruction and ancestral inference from gene-order data. BMC Bioinformatics. 2014;15:354. https://doi.org/10.1186/ s12859-014-0354-6

27. Angiuoli SV, Salzberg SL. Mugsy: fast multiple alignment of closely related whole genomes. Bioinformatics. 2011;27:33442. https:/ / doi.org/10.1093/bioinformatics/btq665

28. Gardner SN, Slezak T, Hall BG. kSNP3.0: SNP detection and phylogenetic analysis of genomes without genome alignment or reference genome. Bioinformatics. 2015; 31:2877-8. https://doi.org/10.1093/bioinformatics/btv271

29. Letunic I, Bork P. Interactive tree of life (iTOL) v3: an online tool for the display and annotation of phylogenetic and other trees. Nucleic Acids Res. 2016;44(W1):W242-5. https:// doi.org/10.1093/nar/gkw290

30. Sealey KL, Harris SR, Fry NK, Hurst LD, Gorringe AR, Parkhill J, et al. Genomic analysis of isolates from the United Kingdom 2012 pertussis outbreak reveals that vaccine antigen genes are unusually fast evolving. J Infect Dis. 2015;212:294-301. https:/ / doi.org/10.1093/infdis/jiu665

31. Block DH, Hussein R, Liang LW, Lim HN. Regulatory consequences of gene translocation in bacteria. Nucleic Acids Res. 2012;40:8979-92. https:/ / doi.org/10.1093/nar/gks694

32. de Gouw D, Hermans PW, Bootsma HJ, Zomer A, Heuvelman K, Diavatopoulos DA, et al. Differentially expressed genes in Bordetella pertussis strains belonging to a ineage which recently spread globally. PLoS One. 2014; 9:e84523. https:// doi.org/10.1371/journal.pone.0084523

33. King AJ, van der Lee S, Mohangoo A, van Gent M, van der Ark A, van de Waterbeemd B. Genome-wide gene expression analysis of Bordetella pertussis isolates associated with a resurgence in pertussis: elucidation of factors involved in the increased fitness of epidemic strains. PLoS One. 2013;8:e66150. https:/ / doi.org/10.1371/journal.pone.0066150

34. Kang Y, Gu C, Yuan L, Wang Y, Zhu Y, Li X, et al. Flexibility and symmetry of prokaryotic genome rearrangement reveal lineage-associated core-gene-defined genome organizational frameworks. MBiol. 2014;5:e01867. https://doi.org/10.1128/ mBio.01867-14

35. Dorman CJ. Genome architecture and global gene regulation in bacteria: making progress towards a unified model? Nat Rev Microbiol. 2013;11:349-55. https://doi.org/10.1038/ nrmicro3007

36. Bryant JA, Sellars LE, Busby SJ, Lee DJ. Chromosome position effects on gene expression in Escherichia coli K-12. Nucleic Acids Res. 2014;42:11383-92. https:/ / doi.org/ 10.1093/nar/gku828

37. Kallonen T, Gröndahl-Yli-Hannuksela K, Elomaa A, Lutyńska A, Fry NK, Mertsola J, et al. Differences in the genomic content of Bordetella pertussis isolates before and after introduction of pertussis vaccines in four European countries. Infect Genet Evol. 2011;11:2034-42. https://doi.org/ 10.1016/j.meegid.2011.09.012

38. West NP, Jungnitz H, Fitter JT, McArthur JD, Guzmán CA, Walker MJ. Role of phosphoglucomutase of Bordetella bronchiseptica in lipopolysaccharide biosynthesis and virulence. Infect Immun. 2000;68:4673-80. https:/ / doi.org/ 10.1128/IAI.68.8.4673-4680.2000

39. Ugalde JE, Czibener C, Feldman MF, Ugalde RA. Identification and characterization of the Brucella abortus phosphoglucomutase gene: role of lipopolysaccharide in virulence and intracellular multiplication. Infect Immun. 2000;68:5716-23. https:/ / doi.org/10.1128/IAI.68.10.57165723.2000 
40. Hardy GG, Magee AD, Ventura CL, Caimano MJ, Yother J. Essential role for cellular phosphoglucomutase in virulence of type 3 Streptococcus pneumoniae. Infect Immun. 2001;69:2309-17. https://doi.org/10.1128/IAI.69.4. 2309-2317.2001

41. Tung SY, Kuo TT. Requirement for phosphoglucose isomerase of Xanthomonas campestris in pathogenesis of citrus canker. Appl Environ Microbiol. 1999;65:5564-70. https:/ / doi.org/10.1128/AEM.65.12.5564-5573.1999

42. Hoffman CL, Gonyar LA, Zacca F, Sisti F, Fernandez J, Wong $\mathrm{T}$, et al. Bordetella pertussis can be motile and express flagellum-like structures. MBiol. 2019;10:e00787-19. https://doi.org/10.1128/mBio.00787-19

43. van Beek LF, de Gouw D, Eleveld MJ, Bootsma HJ, de Jonge MI, Mooi FR, et al. Adaptation of Bordetella pertussis to the respiratory tract. J Infect Dis. 2018;217:198796. https://doi.org/10.1093/infdis/jiy125

44. Wong TY, Hall JM, Nowak ES, Boehm DT, Gonyar LA, Hewlett EL, et al. Analysis of the in vivo transcriptome of Bordetella pertussis during infection of mice. MSphere. 2019; 4:e00154-19. https://doi.org/10.1128/mSphereDirect.00154-19

45. Neo Y, Li R, Howe J, Hoo R, Pant A, Ho S, et al. Evidence for an intact polysaccharide capsule in Bordetella pertussis. Microbes Infect. 2010;12:238-45. https:/ / doi.org/10.1016/ j.micinf.2009.12.002
46. Hoo R, Lam JH, Huot L, Pant A, Li R, Hot D, et al. Evidence for a role of the polysaccharide capsule transport proteins in pertussis pathogenesis. PLoS One. 2014;9:e115243. https://doi.org/10.1371/journal.pone.0115243

47. Ada G, Isaacs D. Carbohydrate-protein conjugate vaccines. Clin Microbiol Infect. 2003;9:79-85. https://doi.org/10.1046/ j.1469-0691.2003.00530.x

48. Fennelly NK, Sisti F, Higgins SC, Ross PJ, van der Heide H, Mooi FR, et al. Bordetella pertussis expresses a functional type III secretion system that subverts protective innate and adaptive immune responses. Infect Immun. 2008;76:1257-66. https://doi.org/10.1128/IAI.00836-07

49. Gaillard ME, Bottero D, Castuma CE, Basile LA, Hozbor D. Laboratory adaptation of Bordetella pertussis is associated with the loss of type three secretion system functionality. Infect Immun. 2011;79:3677-82. https:/ / doi.org/10.1128/ IAI.00136-11

50. Caro V, Bouchez V, Guiso N. Is the sequenced Bordetella pertussis strain Tohama I representative of the species? J Clin Microbiol. 2008;46:2125-8. https://doi.org/10.1128/ JCM.02484-07

Address for correspondence: Branislav Večerek, Institute of Microbiology, Czech Academy of Sciences, Videnska 1083, 14220 Prague 4, Czech Republic; email: vecerek@biomed.cas.cz

\section{EID Podcast}

\section{Tickborne Ehrlichia in North Carolina}

While caring for patients in North Carolina,

Dr. Ross Boyce began to suspect that tickborne Ehrlichia was being underdiagnosed. His study showed that Ehrlichia, despite being relatively

common, was only tested for in about a third of patients thought to have a tickborne illness.

In this EID podcast, Dr. Ross Boyce, an infectious disease physician at the University of North Carolina at Chapel Hill, examines the prevalence and diagnosis of Ehrlichia in North Carolina.

\section{Visit our website to listen: EMERGING https://go.usa.gov/xy6UH INFECTIOUS DISEASES}

